\title{
Characterization of Heart Rate Variability Loss with Aging and Heart Failure Using Sample Entropy
}

\author{
R Goya-Esteban ${ }^{1,2}$, JP Marques de Sá ${ }^{1,3}$, JL Rojo-Álvarez ${ }^{2}$, O Barquero-Pérez ${ }^{1,2}$ \\ ${ }^{1}$ Instituto de Engenharia Biomédica, Porto, Portugal \\ ${ }^{2}$ Universidad Rey Juan Carlos, Fuenlabrada, Madrid, Spain \\ ${ }^{3}$ Universidade do Porto, Porto, Portugal
}

\begin{abstract}
Entropy based measures, such as Sample Entropy (SampEn), have been widely used for quantifying the Heart Rate Variability (HRV) for cardiac risk stratification purposes, with the hypothesis that decreasing entropy points to a perturbation of the complex physiological mechanisms or disease. However, in the literature, higher entropy values have been reported for some pathologies than for healthy subjects, which could be due to the use of a threshold value r tuned relative to the signal standard deviation. In the present paper we apply SampEn to assess the variability of the RR time series from healthy subjects and subjects with Congestive Heart Failure (CHF) in order to discriminate between both groups, as well as to characterize the variability loss due to aging. We conclude that the use of a fixed threshold value $r$ in the SampEn algorithm instead of its conventional setting (as a percentage of the standard deviation of each data series), improves the discrimination capabilities between healthy and $\mathrm{CHF}$ subjects, and it allows to quantify the loss of HRV due to aging in healthy subjects.
\end{abstract}

\section{Introduction}

Given that Heart Rate Variability (HRV) is a relevant marker of autonomic nervous system, numerous attempts to characterize this informative signal have been done. During the last years, a number of nonlinear methods from Chaos Theory, fractal analysis of time series, and Information Theory, have been proposed in this setting. The Approximate Entropy $(A p E n)$, and later the Sample Entropy (SampEn), are in the last group. The former was proposed by Pincus [1] to asses the degree of irregularity in a time series, and it has been widely applied to characterize HRV in adults and fetus [2, 3, 4] with good results. The latter is a modification of the $A p E n$ algorithm that aims to overcome its limitations [5].

However, numerical results obtained in the literature do not always agree with the underlying hypothesis that decreasing entropy points to pathologic states or disorder in the homeostatic regulation. Given that in most of the works using entropy measures the free parameters of the algorithms are set to values previously indicated in the literature, in this work, we studied the influence of such parameters tunning in the assessment of HRV loss due to aging, and in the characterization of HRV in patients affected by Congestive Heart Failure (CHF), having in view a reliable discrimination between healthy and pathological subjects using SampEn.

The structure of the paper is as follows. First, $A p E n$ and SampEn algorithms are introduced. Next, the data sets used and the data analysis by using SampEn are presented. Finally, results and conclusions are presented.

\section{ApEn and SampEn Algorithms}

The characterization of the variability of physiological time series could not be properly achieved by using classic entropy measures such as Kolmogorov-Sinai entropy and its variants, since they may show divergent behavior or they fail to distinguish some processes that appear to differ in complexity [1]. In order to overcome these shortcomings, Pincus formulated a family of statistics for the analysis of short and noisy time series known as $A p E n$.

In order to compute the $A p E n$, the specification of two parameters is previously required: Embedded dimension $m$, that is, the length of the vectors to be compared, and a noise filter threshold $r$.

The procedure for $A p E n$ calculation given $N$ data points $u(1), u(2), \ldots u(N)$ of a signal, is as follows:

- Vector sequences $\mathbf{x}(1), \ldots, \mathbf{x}(N-m+1)$ are obtained, defined by $\mathbf{x}(i)=[u(i), \ldots, u(i+m-1)]$ for $i=1, \ldots, N-$ $m+1$.

- The distance between vectors $\mathbf{x}(i)$ and $\mathbf{x}(j), d[\mathbf{x}(i), \mathbf{x}(j)]$ is defined as the maximum difference, in module, between their respective scalar components, this is

$$
d[\mathbf{x}(i), \mathbf{x}(j)]=\max _{k=1, \ldots, m}(|u(i+k-1)-u(j+k-1)|)
$$


- Based in this distance, the next correlation measurement is defined

$$
C_{i}^{m}(r)=N^{m}(i) /(N-m+1)
$$

where $N^{m}(i)$ counts, for a given vector $\mathbf{x}(i)$, the number of times that $d[\mathbf{x}(i), \mathbf{x}(j)] \leq r$ for $j=1, \ldots, N-m+1$.

- Next, the average of the natural logarithm of $C_{i}^{m}(r)$ is computed for all $i$

$$
\Phi^{m}(r)=\frac{1}{N-m+1} \sum_{i=1}^{N-m+1} \ln C_{i}^{m}(r)
$$

- Finally, ApEn is defined as

$$
\operatorname{ApEn}(m, r)= \begin{cases}\lim _{N \rightarrow \infty} & {\left[\Phi^{m}(r)-\Phi^{m+1}(r)\right] m>0} \\ \lim _{N \rightarrow \infty} & {\left[-\Phi^{1}(r)\right] m=0}\end{cases}
$$

Since in practice $N$ is a finite number, the statistical estimate is computed as

$$
\operatorname{ApEn}(m, r, N)=\left\{\begin{array}{l}
\Phi^{m}(r)-\Phi^{m+1}(r) \quad m>0 \\
-\Phi^{1}(r) \quad m=0
\end{array}\right.
$$

$A p E n$ quantifies the likelihood that runs of patterns that are close for $m$ observations do not remain close at the next incremental comparisons. Therefore, series with repetitive patterns will produce small $A p E n$ values, while less predictable series will produce larger $A p E n$ values.

Several properties of the $A p E n$ make this statistic appropriate for physiological data analysis [3]:

1. There is no need for a large amount of samples to obtain reasonable estimates $\left(30^{m}\right.$ samples are enough).

2 . It is robust to outliers.

3. It is nearly unaffected by noise of magnitude below $r$.

4. Its application is possible for stochastic, deterministic, and mixed processes, because finite values are obtained for all cases.

However, as Pincus himself reported [2], due to the comparison of each template vector with itself to avoid the occurrence of $\ln (0)$ in the algorithm, $A p E n$ is a biased statistic. This bias makes $A p E n$ dependent on the data length, although such effect tends to 0 as $N \rightarrow \infty$. Moreover, ApEn lacks of relative consistency in some cases, although it preserves such relative consistency over a statistically valid range of $(m, r)$ pairs, and hence, the application of this statistic has yielded good results in many studies.

Aiming to improve the $A p E n$ algorithm in order to overcome its limitations, Richman and Moorman [5] developed SampEn statistic, which does not count selfmatches. Additionally, SampEn has two more differences with respect to ApEn: First, only the initial $N-m$ template vectors of length $m$ are considered in both stages of the calculation; Second, it does not use a template approach when estimating conditional probabilities, and hence, the probability measurement is obtained as the natural logarithm of the conditional probability instead of as the ratio of the logarithmic sums.

The SampEn algorithm is computed by the following steps:

- $B_{i}^{m}(r)$ is defined as $(N-m-1)^{-1}$ times the number of template vectors $\mathbf{x}_{m}(j)$ similar to $\mathbf{x}_{m}(i)$ (within $r$ ) where $j=1 \ldots N-m$ with $j \neq i$.

- The average of $B_{i}^{m}(r)$ for all $i$ is calculated as

$$
B^{m}(r)=\frac{1}{N-m} \sum_{i=1}^{N-m} B_{i}^{m}(r)
$$

- Similarly, $A_{i}^{m}(r)$ is defined as $(N-m-1)^{-1}$ times the number of template vectors $\mathbf{x}_{m+1}(j)$ similar to $\mathbf{x}_{m+1}(i)$ (within $r$ ) where $j=1 \ldots N-m$ with $j \neq i$.

- The average of $A_{i}^{m}(r)$ for all $i$ is calculated as

$$
A^{m}(r)=\frac{1}{N-m} \sum_{i=1}^{N-m} A_{i}^{m}(r)
$$

- $\operatorname{SampEn}(m, r)$ and its estimated statistic $\operatorname{SampEn}(m, r, N)$ are defined as follows

$$
\begin{gathered}
\operatorname{SampEn}(m, r)=\lim _{N \rightarrow \infty}\left\{-\ln \left[A^{m}(r) / B^{m}(r)\right]\right\} \\
\operatorname{SampEn}(m, r, N)=-\ln \left[A^{m}(r) / B^{m}(r)\right]
\end{gathered}
$$

SampEn exhibits higher independence of the data length, as well as relative consistency in more situations than $A p E n$ does. It also maintains the original features that make $A p E n$ appropriate for the study of physiological signals.

\section{Dataset and SampEn Analysis}

SampEn statistic was used to assess the variability of the RR-interval signals from 24-hour Holter recordings in healthy subjects and in subjects with CHF. Both sets of recordings were obtained from Physionet database [6]. The data were obtained from recordings of 72 healthy subjects, aged from 20 to 76 years old, and 44 subjects affected by $\mathrm{CHF}$, from 22 to 79 years old. All datasets were filtered to exclude artifacts, missed detections and isolated ectopic beats. Furthermore, RR intervals lower than 200 $\mathrm{ms}$ and greater than $2000 \mathrm{~ms}$ were excluded, as well as those which differed more than $20 \%$ from the previous and the subsequent RR intervals [7].

Given that the free parameter setting in entropy methods is crucial for their performance, we first studied the influence of the free parameters tuning in the performance of the statistic in order to maximize the separation between 
healthy and pathologic subjects. For this purpose, we evaluated a set of possible values for $m=1,2,3$. Concerning to threshold value $r$, SampEn was computed according to two methods:

1. Parameter $r$ set to a certain percentage of the standard deviation (SD) of each data series, which is the method conventionally used in the literature [2, 5, 8] (using $r=$ $[0.1,0.15,0.2] * S D)$.

2. Parameter $r$ set to a percentage of the mean standard deviation of whole recordings (from healthy and CHF subjects), which means a fixed $r$ for all the recordings (using $r=10,15,20)$.

This last method is justified in [4], where better results were reported in Fetal Heart Rate (FHR) Variability assessment with $r$ independent of the SD of each recording.

The discrimination capabilities of the statistics to distinguish between the young and the elderly groups were also studied, by splitting the recordings from healthy subjects into two groups (young group, from 20 to 50 years, and elderly group, from 51 to 80 years).

Finally, we split the recordings from healthy subjects into six different groups according to the age of the subjects, and SampEn was computed for each group in order to analyze the evolution of the statistic. The relation of the statistic with the age was analyzed using linear regression of the SampEn vs. age, and then obtaining the slope (variation vs. year index) and its determination coefficient, in this case both for healthy and for CHF subjects.

\section{4. $\quad$ Results}

Table 2 shows the combination of $m$ and $r$ parameters that achieve larger discrimination, for each method of setting the filter threshold. We found that the use of a fixed threshold value $r$, obtained by method 2, instead of the more widely extended tuning of $r$ as a percentage of the SD of each data series, yielded better discrimination between healthy and CHF subjects (lower p-values in the Student's t test). Also, higher SampEn values were obtained for healthy subjects than for CHF subjects, which is in agreement with the idea of loss of HRV in pathological conditions, whereas, for $r$ being a percentage of the SD of each data series, higher SampEn values for CHF subjects than for healthy subjects were obtained. The reason for this result could be that the subjects with $\mathrm{CHF}$ have lower SD than the healthy ones (see Table 1), and when $r$ is set dependent on the SD of each recording, healthy recordings are scaled by higher values than $\mathrm{CHF}$ subjects, which does not only affect the amplitude of the data, but also the relative difference between the samples.

For young and elderly groups, the combination of $m$ and $r$ parameters that achieve larger discrimination for each method is shown in Table 3. In this case, both methods provide similar discrimination capabilities and higher val-
Table 1. Standard deviation of the different groups. Mean $\pm S D$.

\begin{tabular}{lc}
\hline \hline & SD \\
\hline Healthy & $136.84 \pm 32.67$ \\
Healthy-Young & $136.20 \pm 35.51$ \\
Healthy-Elderly & $137.20 \pm 31.36$ \\
CHF & $66.98 \pm 38.87$ \\
\hline \hline
\end{tabular}

Table 2. Mean $\pm S D$ of SampEn for both methods of setting $r$. Symbol * means significant variation $(p<0.001)$ between healthy and CHF subjects.

\begin{tabular}{lcc}
\hline \hline & SampEn(3,15) & SampEn(1,0.1SD) \\
\hline Healthy & $1.00 \pm 0.23$ & $1.09 \pm 0.27$ \\
CHF & $0.51 \pm 0.26^{*}$ & $1.37 \pm 0.36^{*}$ \\
\hline \hline
\end{tabular}

ues for young than for elderly subjects, the reason is that the standard deviations for both groups are very similar (Table 1), and hence in method 1, the SD does not interfere in the computation of the irregularity degree. We also found that, with $r$ set by method 2, it was possible to quantify the loss of HRV due to the aging in healthy subjects, which was less clearly present with $r$ set by method 1 , since it did not show a fixed increasing or decreasing trend. Table 4 and Figure 1 show the results for each age group. The linear regression (see Table 5) shows that for $\mathrm{CHF}$ subjects no correlation was found between the age and the variation of SampEn values.

Table 3. Mean $\pm S D$ of SampEn for both methods of setting $r$. Symbol * means significant variation $(p<0.001)$ between young and elderly subjects.

\begin{tabular}{lcc}
\hline \hline & SampEn(2,20) & SampEn(2,0.2SD) \\
\hline Young & $0.87 \pm 0.23$ & $0.66 \pm 0.18$ \\
Elderly & $0.61 \pm 0.13^{*}$ & $0.43 \pm 0.14^{*}$ \\
\hline \hline
\end{tabular}

Table 4. SampEn evolution with age. Mean $\pm S D$ for both methods of setting $r$.

\begin{tabular}{lcc}
\hline \hline Age(years) & SampEn(2,20) & SampEn(2,0.2SD) \\
\hline $20-30$ & $0.91 \pm 0.29$ & $0.61 \pm 0.13$ \\
$31-40$ & $0.86 \pm 0.21$ & $0.68 \pm 0.21$ \\
$41-50$ & $0.76 \pm 0.24$ & $0.63 \pm 0.20$ \\
$51-60$ & $0.67 \pm 0.16$ & $0.58 \pm 0.07$ \\
$61-70$ & $0.61 \pm 0.15$ & $0.42 \pm 0.14$ \\
$71-80$ & $0.54 \pm 0.20$ & $0.47 \pm 0.16$ \\
\hline \hline
\end{tabular}




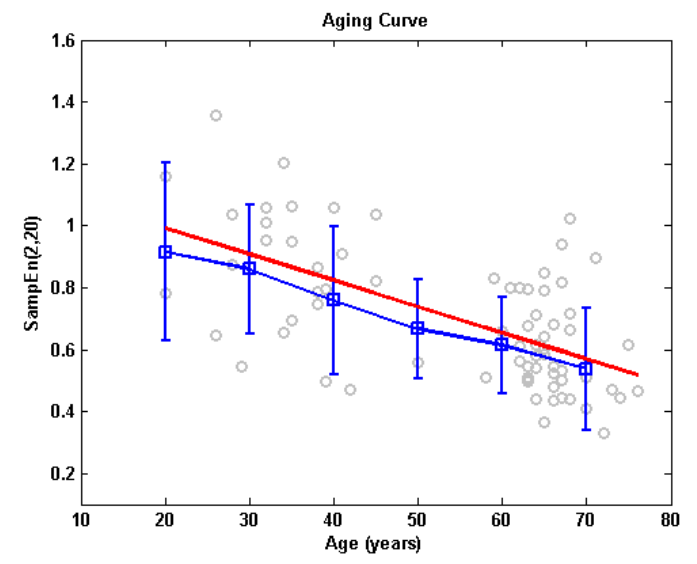

(a)

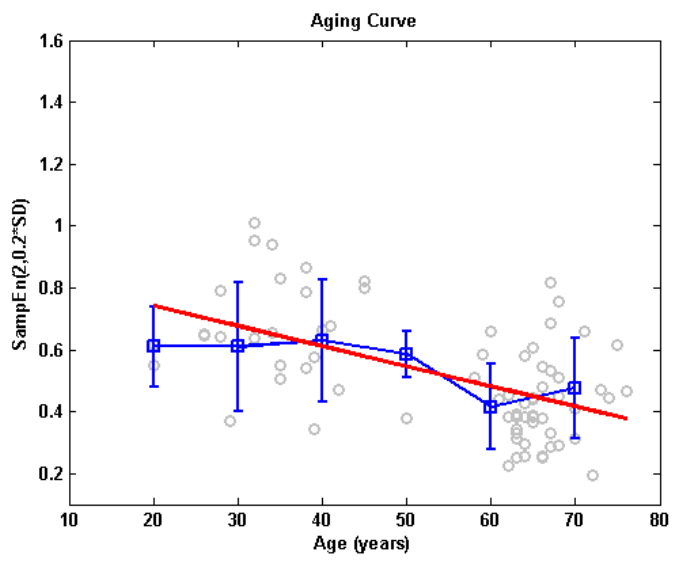

(b)

Figure 1. Evolution of SampEn with age. Circles represent the SampEn for each subject, the squares the mean of each age group, the bars the standard deviation of each age group, and the straight line represents the fitted regression line. (a) For fixed $r$, set by method 2. (b) For $r$ as a percentage of each data series standard deviation.

Table 5. Results of the linear regression of SampEn vs. age. Symbol * means significant $(p<0.001)$.

\begin{tabular}{lcc}
\hline \hline & Index var/year & $r^{2}$ \\
\hline Healthy & & \\
SampEn(2,20) & -0.0085 & $0.3653^{*}$ \\
SampEn(2,0.2SD) & -0.0065 & $0.2935^{*}$ \\
CHF & & \\
SampEn(2,20) & -0.0011 & 0.0036 \\
SampEn(2,0.2SD) & -0.0125 & 0.1857 \\
\hline \hline
\end{tabular}

\section{Conclusions}

By using a fixed threshold value $r$ in the SampEn algorithm, we were able to obtain a good representation of the HRV loss for CHF condition as well as a steady decrease of the HRV-SampEn with aging. Moreover, higher discrimination between healthy and CHF subjects was achieved, and a usable aging curve of HRV loss could be built for healthy subjects. For this last purpose, further studies with larger number of recordings are needed.

\section{Acknowledgements}

This work has been partly supported by Research Grant TEC-2007-68096-C02-TCM from Spanish Ministry of Science and Technology.

\section{References}

[1] Pincus SM. Approximate entropy as a measure of system complexity. Proc Natl Acad Sci 1991;88:2297-2301.
[2] Pincus SM, Goldberger AL. Physiological time-series analysis: what does regularity quantify? Am J Physiol Heart Circ Physiol 1994;35:1643-1656.

[3] Pincus SM. Assesing serial irregularity and its implications for health. Annals New York Academy of Sciences 2001; 954:245-267.

[4] Marques-de S JP. Characterization of fetal heart rate using approximate entropy. IEEE Computers in Cardiology 2005; 32:671-673.

[5] Richman JS, Moorman JR. Physiological time-series analysis using approximate entropy and sample entropy. Am J Physiol Heart Circ Physiol 2000;278:2039-2049.

[6] Goldberger AL, Amaral LAN, Glass L, Hausdorff JM, Ivanov PC, Mark RG, Mietus JE, Moody GB, Peng CK, Stanley HE. PhysioBank, PhysioToolkit, and PhysioNet: Components of a new research resource for complex physiologic signals. Circulation 2000 (June 13);101(23):e215e220.

[7] Malik M, Cripps T, Farrell T, Camm A. Prognostic value of heart rate variability after myocardial infarction. a comparison of different data-processing methods. Medical and Biological Engineering and Computing 1989;27:603-621.

[8] Signorini M, Ferrario M, Marchetti M, Marseglia A. Nonlinear analysis of heart rate variability signal for the characterization of cardiac heart failure patients. Conf Proc IEEE Eng Med Biol Soc 2006;1(1):3431-3434.

Address for correspondence:

Rebeca Goya-Esteban

Universidad Rey Juan Carlos

Camino del Molino s/n, Fuenlabrada-28943, Madrid (Spain)

rebegoya@gmail.com 